\title{
Kleines Einmaleins der Pflege
}

Liebe Leserinnen, liebe Leser

Stürzen, Thrombosen, Kontrakturen oder Pneumonien vorbeugen: Prophylaxen gelten als Kleines Einmaleins der Pflege, schreibt Siegfried Huhn in dieser NOVAcura. Dank ihnen wird das Risiko für pflegerische und medizinische Komplikationen kleiner und die Gesundheit Pflegebedürftiger bleibt erhalten bzw. ihre gesundheitlichen Probleme werden nicht gravierender. Deshalb müssen alle Fachpersonen und pflegende An- und Zugehörige über die notwendigen prophylaktischen Massnahmen Bescheid wissen.

Sich theoretisches und praktisches Wissen über diese Massnahmen anzueignen und ggf. auch zu vermitteln, liegt in der Verantwortung jeder Pflegefachperson. Ihr Wissen muss sich von anekdotischem und rein überliefertem Wissen unterscheiden, auf aktuellen theoretischen Grundlagen basieren und wissenschaftlich fundiert sein. Oft fehlt bei einer durchgeführten Pflegehandlung jedoch diese Grundlage oder die wissenschaftlich bestätigte Wirksamkeit. Geht es um Prophylaxen, müssen sich Fachpersonen also immer wieder auch mit Fragen über evidenzbasiertes Pflegehandeln auseinandersetzen (nach Siegfried Huhn, beide Beiträge in diesem Heft).

Das zeigt einmal mehr, wie hoch die Anforderungen für den Pflegeberuf sind. Das wird nicht nur in Siegfried Huhns Beiträgen deutlich, sondern auch in allen weiteren. Joëlle Riedweg, Leonie Kammer, Silvia Thomann \& Niklaus Bernet zeigen, dass in Schweizer Spitälern bei sturz- und dekubitusgefährdeten Personen präventive Massnahmen gut angewendet werden, bei der Identifizierung und dem aktiven Einbezug von Risikopatientinnen und -patienten aber noch Entwicklungspotential besteht. Hardy-Thorsten Panknin o Stefan Schröder stellen eine aktuelle Studie vor, die untersucht, ob die oft verwendete Braden-Skala sich auch für die Vorhersage des Dekubitusrisikos von Covid-19 betroffenen Intensivpatientinnen und -patienten eignet, während Eva Horvath ausführt, wie wichtig es für die Langzeitpflege ist, vorhandene Konzepte aus dem Akut-

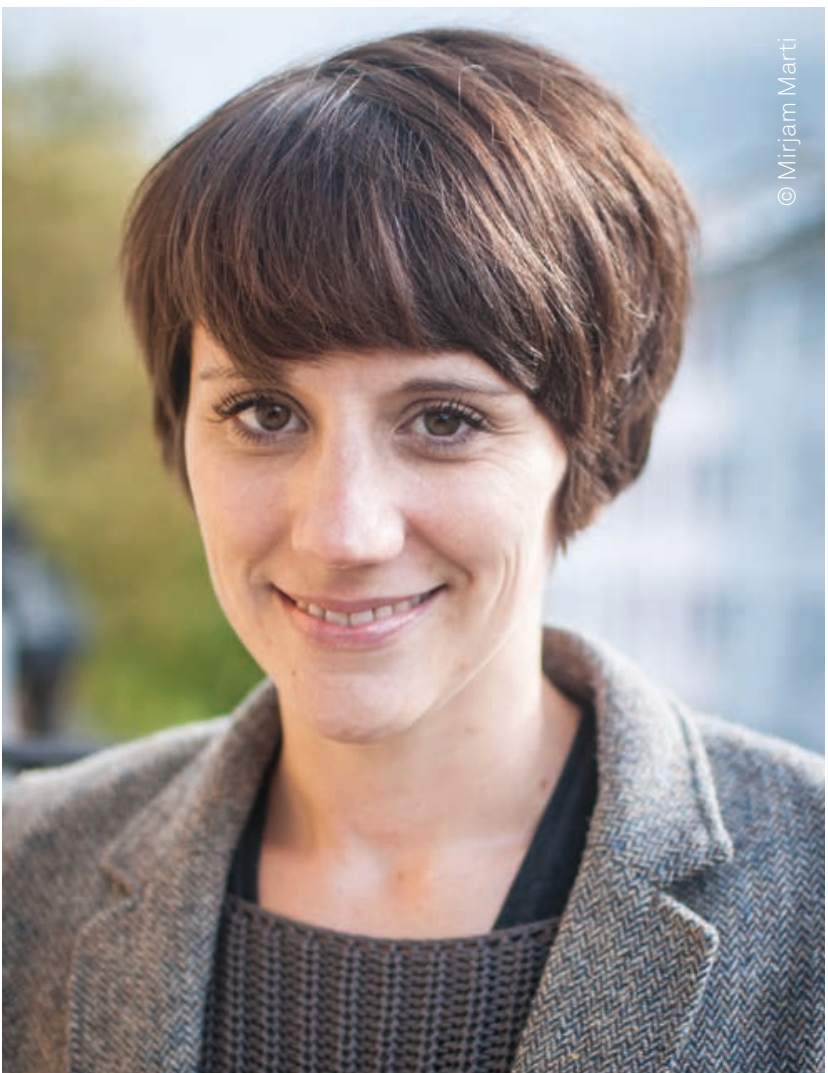

bereich nicht einfach zu übernehmen, sondern entsprechend zu adaptieren. Sylke Werner zeigt, wie sich prophylaktische Massnahmen in den geriatrischen Pflegealltag integrieren lassen, und Elke Steudter schreibt über das notwendige kritische Denken in der Pflege: Wie können Pflegende gute Entscheidungen darüber treffen, welche Handlungen wann wie ausgeführt werden sollen?

Ich wünsche Ihnen eine interessante Lektüre und grüsse Sie herzlich.

Eveline Kühni 


\section{Inhalt}

Editorial

Kleines Einmaleins der Pflege

Eveline Kühni

Schwerpunkt

Professionell und nachvollziehbar entscheiden

Elke Steudter

Pflegewissen und Prophylaxen

Siegfried Huhn

Schutz vor Sturz und Dekubitus

Joëlle Riedweg, Leonie Kammer, Silvia Thomann \& Niklaus Bernet

Dekubituskonzept in der Langzeit und Übergangspflege

Eva Horvath

Kontrakturprophylaxe

Siegfried Huhn

Medientipps zum Thema

Lena-Marie Wimmel

Folkus

Psychiatrische Pflegefachpersonen befassen sich mit psychischen

29 Auswirkungen der Pandemie

Psychiatriekommission des Schweizer Berufsverbands der

Pflegefachfrauen und Pflegefachmänner SBK

\begin{tabular}{llr}
\hline Palliative Care & $\begin{array}{l}\text { „Ich bin meinem Gefühl gefolgt“ } \\
\text { Stefan Müller }\end{array}$ & $\mathbf{3 3}$ \\
\hline Pflegen zu Hause & $\begin{array}{l}\text { Vorbeugen, bevor es zu spät ist! } \\
\text { Sylke Werner }\end{array}$ & $\mathbf{3 5}$ \\
\hline Bildung & Pflege und Forschung & $\mathbf{4 0}$ \\
& Brigitte Teigeler & $\mathbf{4 2}$ \\
& Dekubitusrisiko bei Intensivpatienten & \\
& Hardy-Thorsten Panknin \& Stefan Schröder & $\mathbf{4 5}$ \\
& Ein Modell mit menschlichen Zügen \\
& Monika Bachmann & $\mathbf{4 9}$ \\
\hline Dementia Care & Demenz und Ernährung \\
& Lee Martin & $\mathbf{5 3}$ \\
\hline à propos & & $\mathbf{5 4}$ \\
\hline Vorschau/Termine & $\mathbf{5 5}$
\end{tabular}

